\title{
Self-assembled microporous M-HOFs based on octahedral rhenium cluster with benzimidazole
}

Dmitry I. Konovalovt, Anton A. Ivanovt*, Yuri A. Vorotnikovt, Natalia V. Kuratievat, Ilia V. Eltsov $\ddagger$ Konstantin A. Kovalenkot, Michael A. Shestopalov ${ }^{\dagger}$

†Nikolaev Institute of Inorganic Chemistry SB RAS, 3 Acad. Lavrentiev Ave., 630090 Novosibirsk, Russian Federation

¥Novosibirsk State University, 2 Pirogova Str., 630090 Novosibirsk, Russian Federation 


\section{TABLE OF CONTENT}

FTIR spectroscopy 3

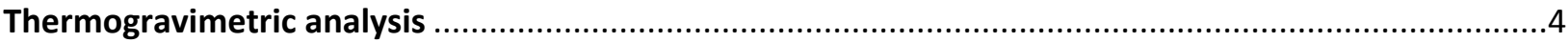

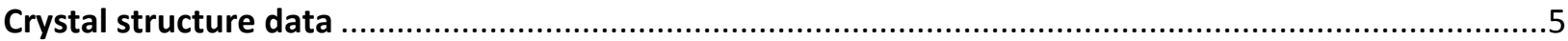

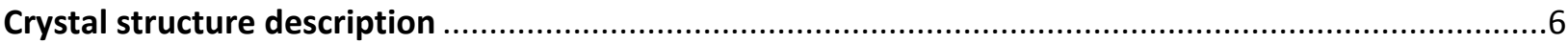

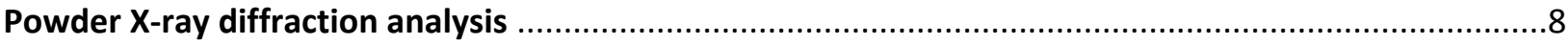

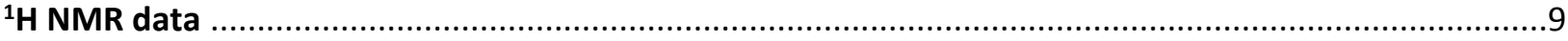

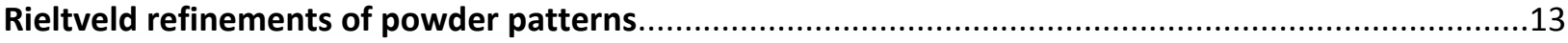

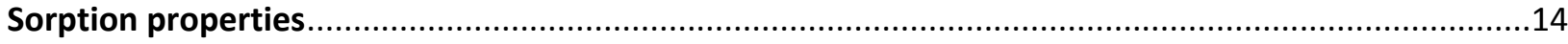

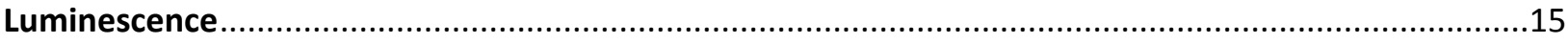

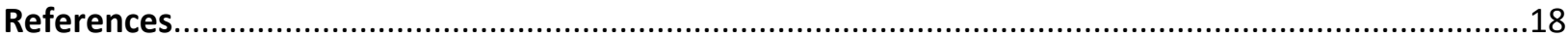




\section{FTIR spectroscopy}

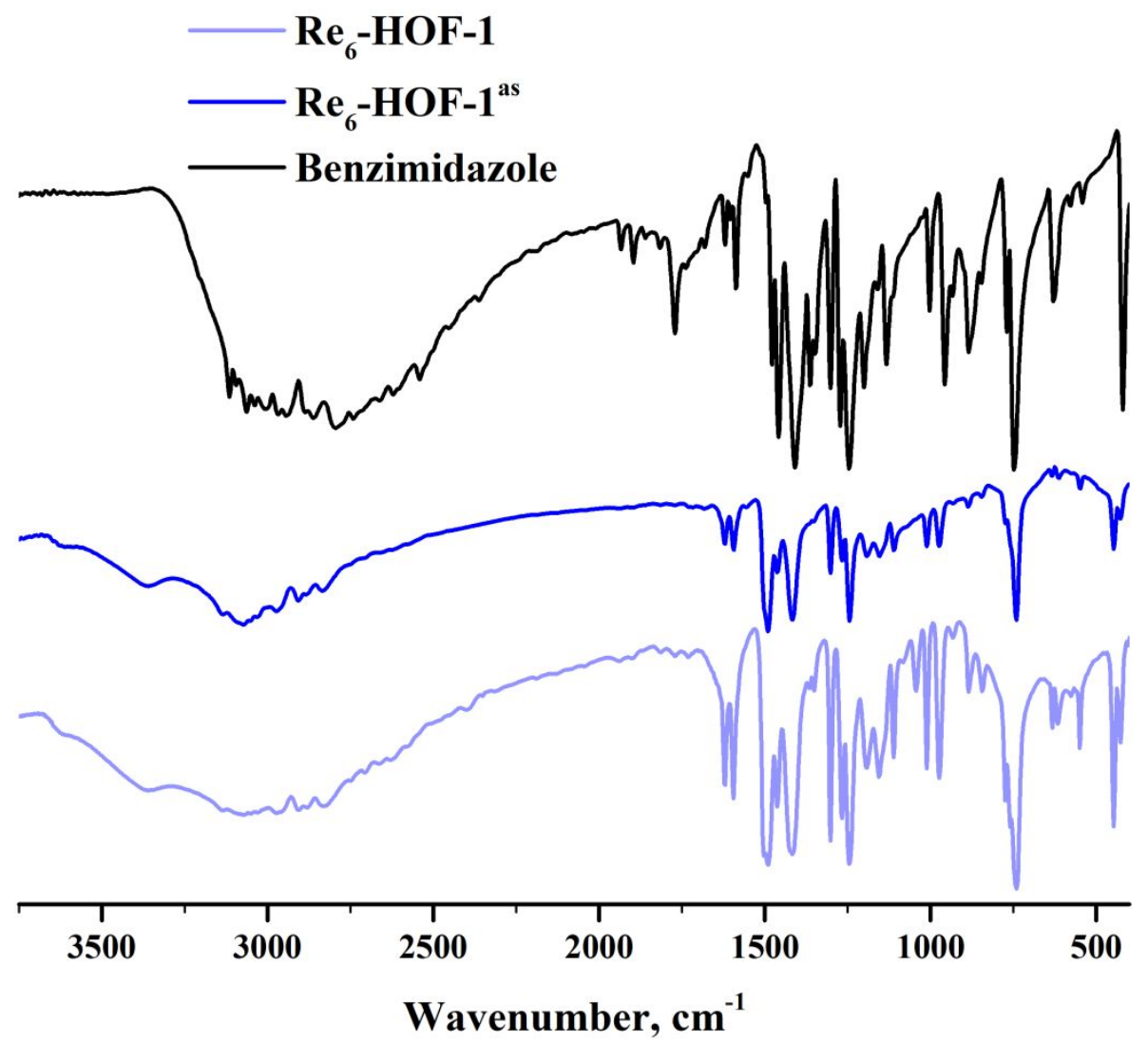

Figure S1. FTIR spectra of $\mathbf{R e}_{6}-\mathrm{HOF}-\mathbf{1}^{\text {as }}$ and $\mathbf{R e}_{6}-\mathrm{HOF}-\mathbf{1}$ in compassion with benzimidazole.

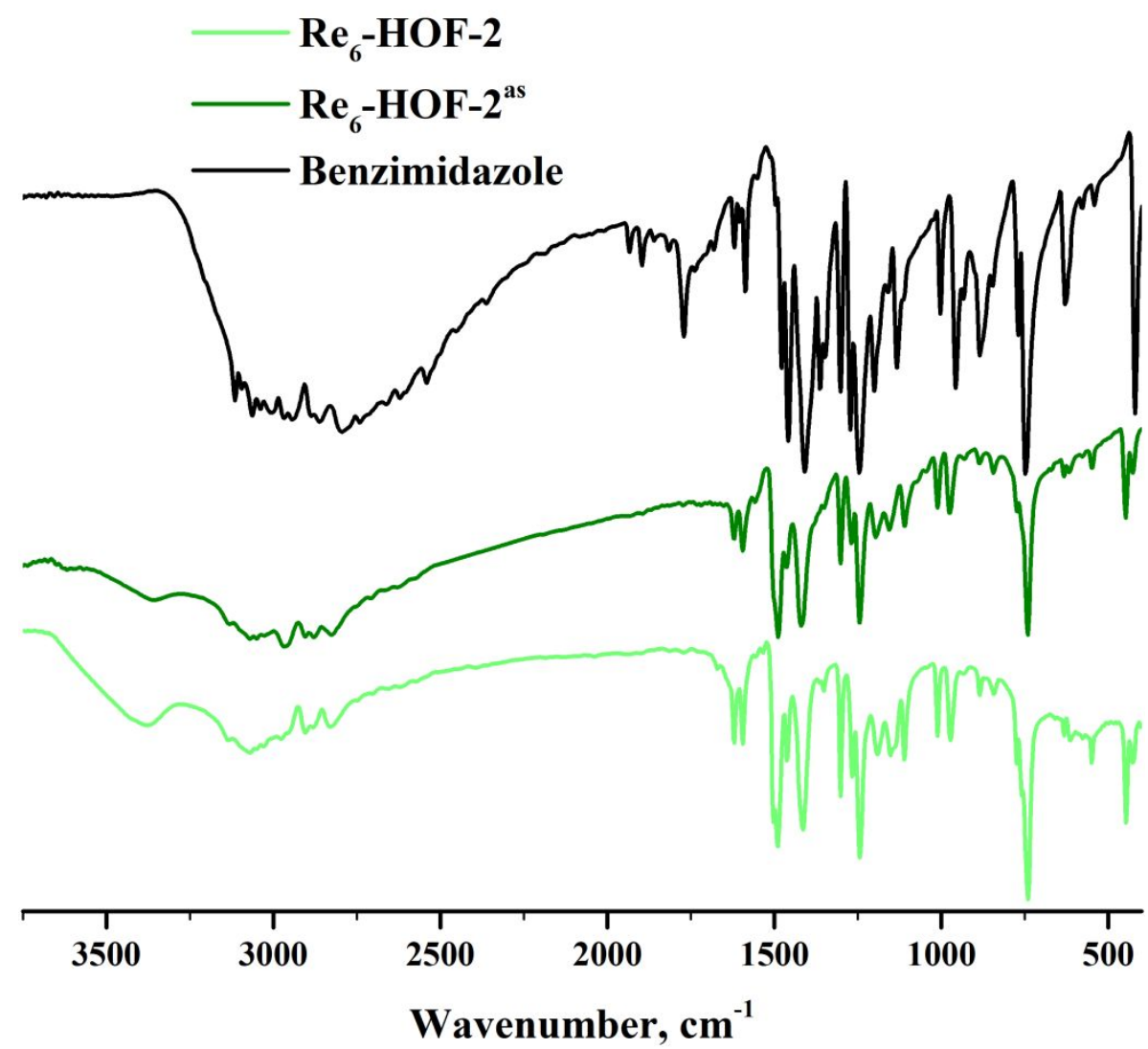

Figure S2. FTIR spectra of $\mathbf{R e}_{6}-\mathrm{HOF}-\mathbf{2}^{\text {as }}$ and $\mathbf{R e}_{6}-\mathrm{HOF}-\mathbf{2}$ in compassion with benzimidazole. 
Thermogravimetric analysis

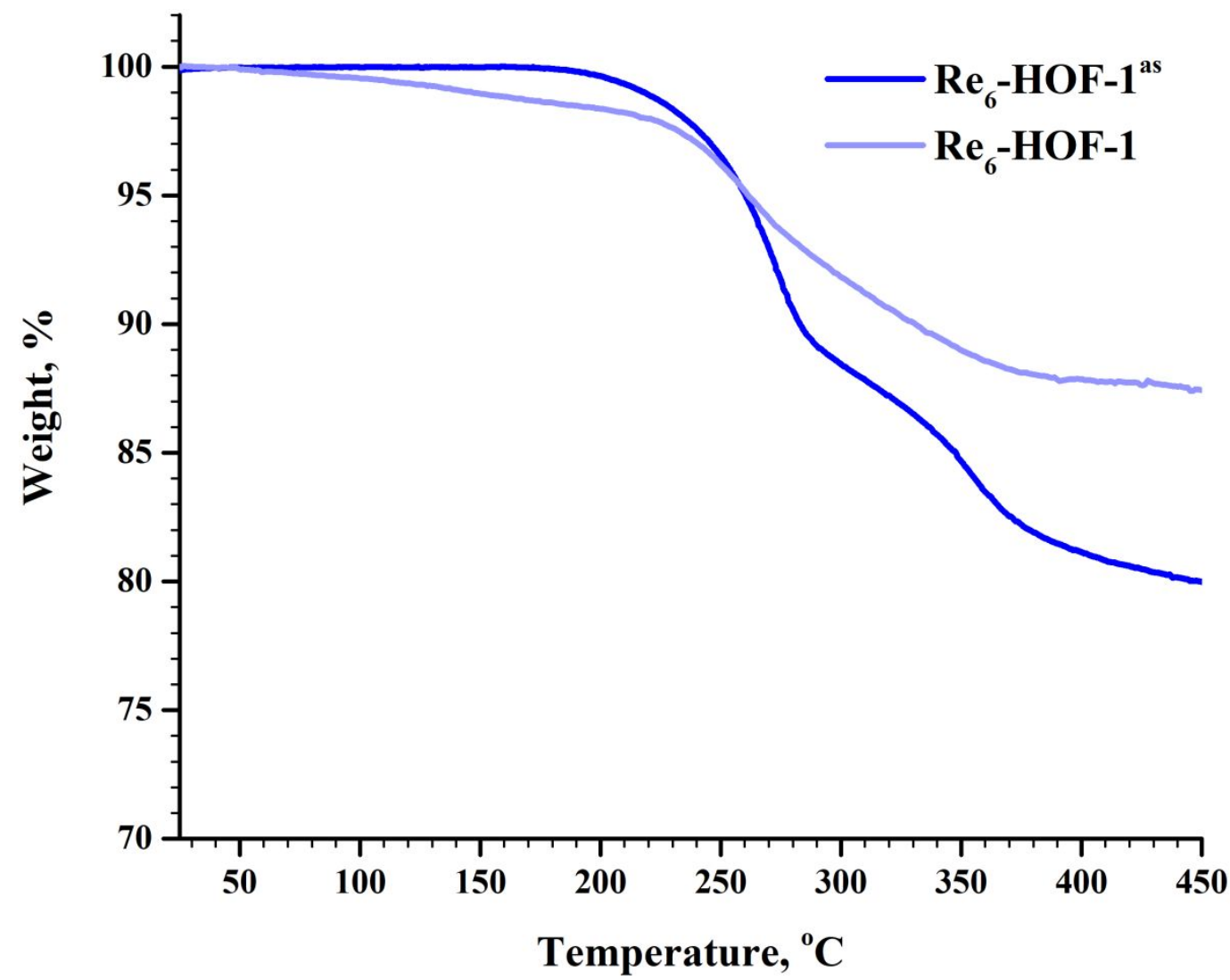

Figure S3. TGA curves of $\mathbf{R e}_{6}-\mathrm{HOF}-\mathbf{1}^{\text {as }}$ and $\mathbf{R e}_{6}-\mathrm{HOF}-1$. Heating rates of $10^{\circ} \mathrm{C} \cdot \mathrm{min}^{-1}$.

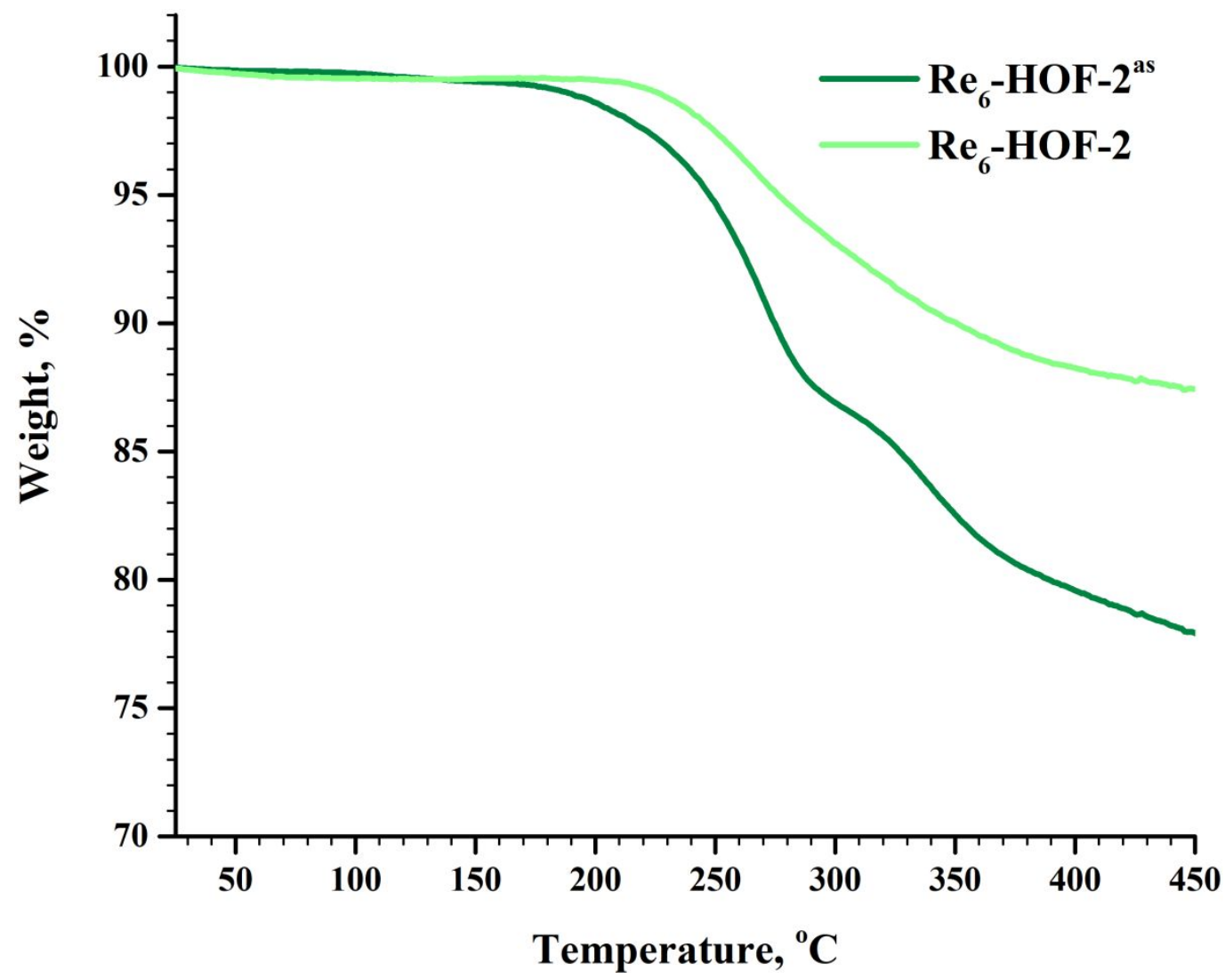

Figure S4. TGA curves of $\mathbf{R e}_{6}-\mathrm{HOF}-2^{\text {as }}$ and $\mathbf{R e}_{6}-\mathrm{HOF}-2$. Heating rates of $10^{\circ} \mathrm{C} \cdot \mathrm{min}^{-1}$. 


\section{Crystal structure data}

Table S1. Crystal data, data collection and refinement parameters for $\left[\left\{\mathrm{Re}_{6} \mathrm{Se}_{8}{ }_{8}\right\}(\mathrm{bimzH})^{\mathrm{a}}{ }_{6}\right] \mathrm{Cl}_{2}\left(\mathrm{Re}_{6}{ }^{-}\right.$ HOF-1 $\left.{ }^{\text {as }}\right)$ and $\left[\left\{\mathrm{Re}_{6} \mathrm{Se}_{8}{ }_{8}\right\}\left(\right.\right.$ bimzH) $\left.{ }_{6}{ }_{6}\right] \mathrm{Br}_{2}\left(\mathbf{R e}_{6}\right.$-HOF-2as).

\begin{tabular}{|c|c|c|}
\hline Compound & $\operatorname{Re}_{6}-\mathrm{HOF}-1^{\text {as }}$ & $\mathrm{Re}_{6}-\mathrm{HOF}-2^{\text {as }}$ \\
\hline Empirical formula & $\mathrm{C}_{42} \mathrm{H}_{36} \mathrm{Cl}_{2} \mathrm{~N}_{12} \mathrm{Re}_{6} \mathrm{Se}_{8}$ & $\mathrm{C}_{42} \mathrm{H}_{36} \mathrm{Br}_{2} \mathrm{~N}_{12} \mathrm{Re}_{6} \mathrm{Se}_{8}$ \\
\hline Formula weight & 2528.61 & 2617.53 \\
\hline Temperature, $\mathrm{K}$ & $150(2)$ & $150(2)$ \\
\hline Crystal system & Triclinic & Triclinic \\
\hline Space group & $P \overline{1}$ & $P \overline{1}$ \\
\hline$a, \AA$ & $13.4191(6)$ & $13.3724(5)$ \\
\hline$b, \AA$ & $13.4205(6)$ & $13.4544(5)$ \\
\hline$c, \AA$ & $13.4212(6)$ & $13.5139(4)$ \\
\hline$\alpha,{ }^{\circ}$ & $66.4710(10)$ & $67.9810(10)$ \\
\hline$B,^{\circ}$ & $66.3830(10)$ & $65.6170(10)$ \\
\hline$r_{1}^{\circ}$ & $66.5180(10)$ & $69.2340(10)$ \\
\hline$V, \AA^{3}$ & $1946.90(15)$ & 1994.71(12) \\
\hline$Z$ & 1 & 1 \\
\hline$\rho_{\text {calc }}, \mathrm{g} / \mathrm{cm}^{3}$ & 2.157 & 2.179 \\
\hline$\mu, \mathrm{mm}^{-1}$ & 13.127 & 13.747 \\
\hline$F(000)$ & 1128 & 1164 \\
\hline Crystal size & $0.12 \times 0.12 \times 0.06$ & $0.1 \times 0.08 \times 0.04$ \\
\hline \multirow[t]{2}{*}{$\begin{array}{l}2 \Theta \text { range for data } \\
\text { collection, }^{\circ}\end{array}$} & 1.726 to 27.634 & 2.675 to 26.791 \\
\hline & $-17 \leq h \leq 17$ & $-16 \leq h \leq 16$ \\
\hline \multirow[t]{2}{*}{ Index ranges } & $-17 \leq k \leq 17$ & $-17 \leq k \leq 17$ \\
\hline & $-17 \leq I \leq 17$ & $-16 \leq I \leq 17$ \\
\hline Reflections collected & 19148 & 16391 \\
\hline Independent reflections & $8936\left[R_{\text {int }}=0.0264\right]$ & $8379\left[R_{\text {int }}=0.0399\right]$ \\
\hline Data/restraints/parameters & 8936 / 0 / 316 & 8379 / 0 / 316 \\
\hline Goodness-of-fit on $F^{2}$ & 1.088 & 1.108 \\
\hline$R_{1} / w R_{2}(I>2 \sigma(I))$ & $0.0621 / 0.1817$ & $0.0467 / 0.1382$ \\
\hline$R_{1} / w R_{1}$ (all data) & $0.0968 / 0.2059$ & $0.0798 / 0.1498$ \\
\hline$\Delta \rho_{\max } / \Delta \rho_{\min }\left(\mathrm{e} \cdot \AA^{-3}\right)$ & $10.308 /-2.810$ & $2.306 /-1.866$ \\
\hline
\end{tabular}




\section{Crystal structure description}

Table S2. Main interatomic distances $(\AA ̊)$ for $\mathbf{R e}_{6}-\mathrm{HOF}-\mathbf{1}^{\text {as }}$ and $\mathbf{R e}_{6}-\mathrm{HOF}-\mathbf{2}^{\text {as }}$ in comparison with literature data.

\begin{tabular}{|c|c|c|c|c|}
\hline Compound & $\operatorname{Re}-\operatorname{Re}$ & $\mathrm{Re}-\mathrm{Se}$ & $\mathrm{Re}-\mathrm{N}$ & Ref \\
\hline $\mathrm{Re}_{6}-\mathrm{HOF}-\mathbf{1}^{\text {as }}$ & $\begin{array}{l}2.6126(6)- \\
2.6177(6)\end{array}$ & $\begin{array}{c}2.5282(12)- \\
2.5352(11) \\
\end{array}$ & $\begin{array}{c}2.165(9)- \\
2.177(8) \\
\end{array}$ & This study \\
\hline $\mathrm{Re}_{6}-\mathrm{HOF} 2^{\text {as }}$ & $\begin{array}{l}2.6160(6)- \\
2.6202(6)\end{array}$ & $\begin{array}{c}2.5169(12)- \\
2.5246(12)\end{array}$ & $\begin{array}{c}2.138(9)- \\
2.172(8)\end{array}$ & This study \\
\hline$\left[\left\{\mathrm{Re}_{6} \mathrm{Se}_{8}\right\}(\mathrm{pzH})_{6}\right] \mathrm{Cl}_{2}$ & $\begin{array}{r}2.6062(6)- \\
2.6293(6)\end{array}$ & $\begin{array}{c}2.5031(10)- \\
2.5381(10)\end{array}$ & $\begin{array}{c}2.153(8)- \\
2.185(7)\end{array}$ & [1] \\
\hline$\left[\left\{\mathrm{Re}_{6} \mathrm{Se}_{8}\right\}(\mathrm{imzH})_{6}\right] \mathrm{Cl}_{2} \cdot 4 \mathrm{DMSO}$ & $\begin{array}{c}2.6163(6)- \\
2.6248(6) \\
\end{array}$ & $\begin{array}{c}2.5205(12)- \\
2.5309(12) \\
\end{array}$ & $\begin{array}{c}2.166(8)- \\
2.182(8) \\
\end{array}$ & [2] \\
\hline $\begin{array}{c}\mathrm{K}_{4}\left[\left\{\mathrm{Re}_{6} \mathrm{Se}_{8}\right\}(1,2,3-\operatorname{trz})_{6}\right] \\
\cdot 6(1,2,3-\operatorname{trzH})\end{array}$ & $\begin{array}{l}2.6152(1)- \\
2.6157(1)\end{array}$ & $\begin{array}{l}2.5197(1)- \\
2.5247(1)\end{array}$ & $2.1553(1)$ & [3] \\
\hline
\end{tabular}

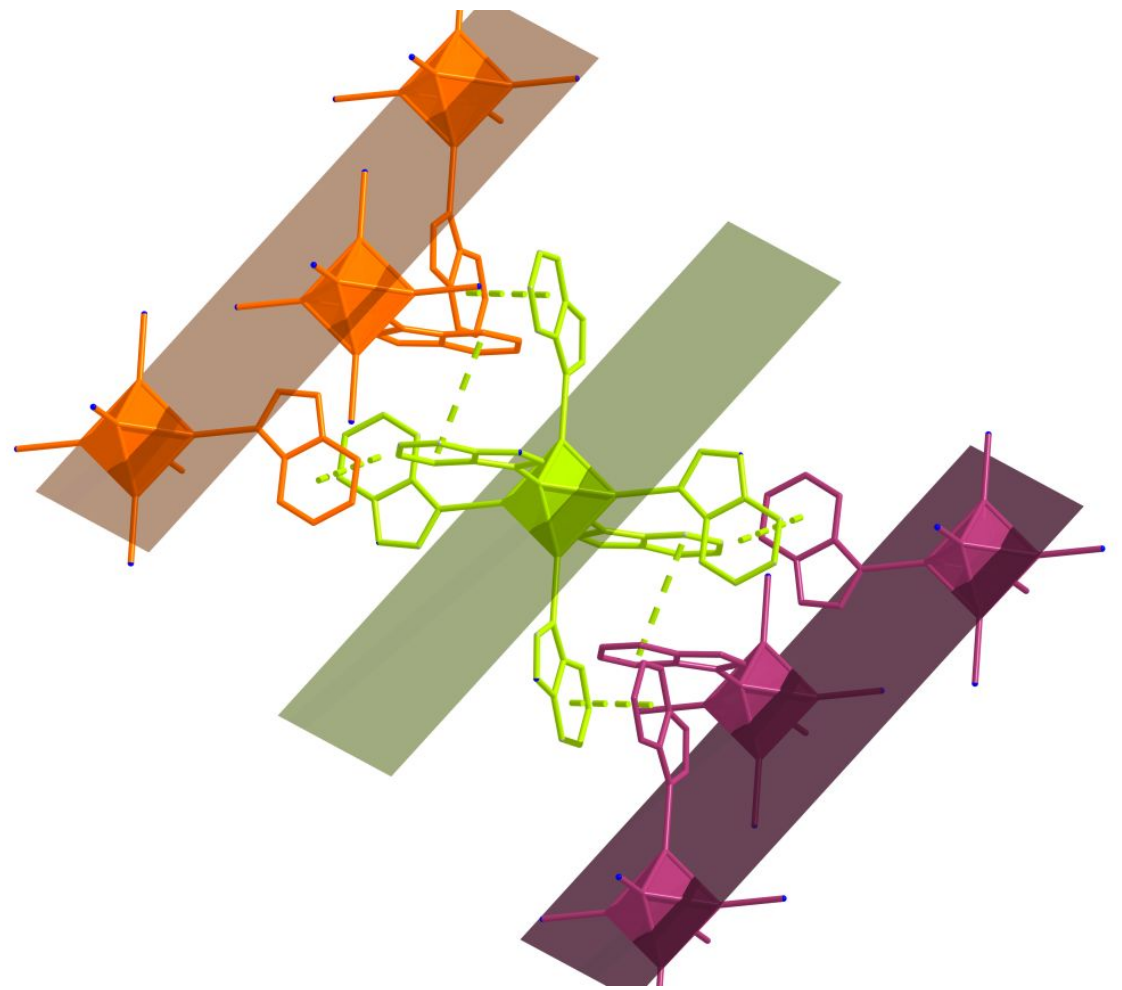

Figure S5. $\pi-\pi$ stacking between adjacent layers observed in structure of $\mathrm{Re}_{6}-\mathrm{HOFs}$. 


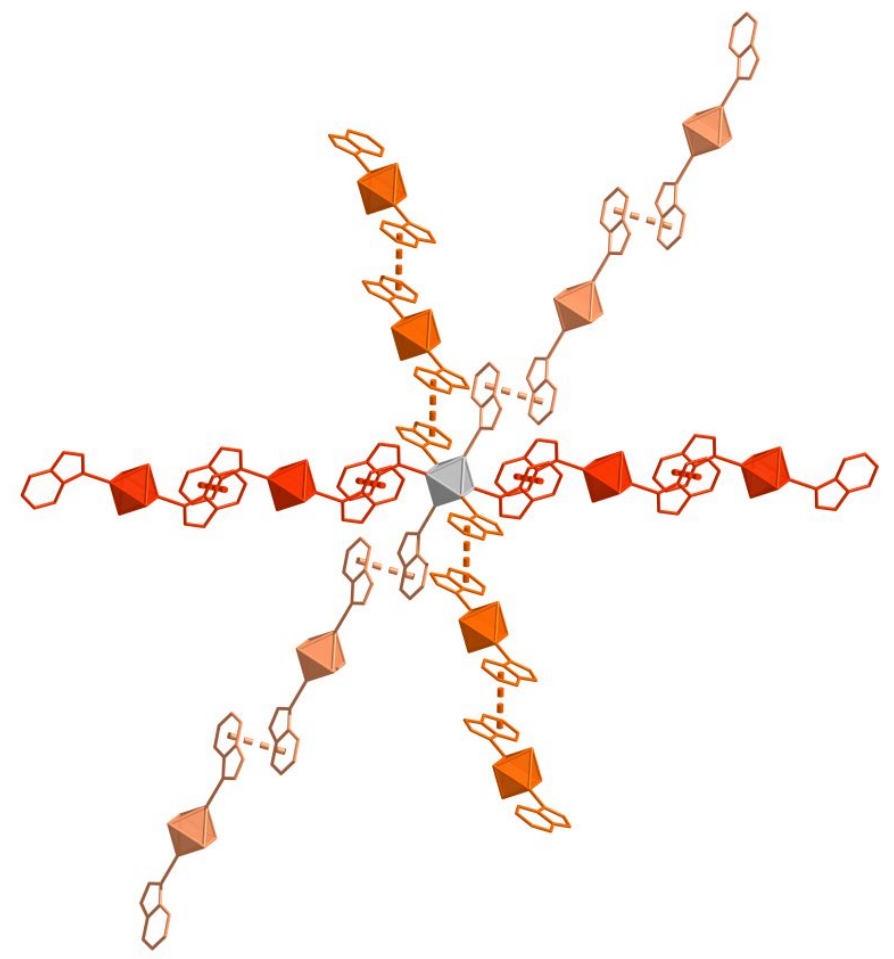

Figure S6. Chains formed by $\pi-\pi$ stacking interactions observed in structure of $\operatorname{Re}_{6}-\mathrm{HOFs}$. 


\section{Powder X-ray diffraction analysis}

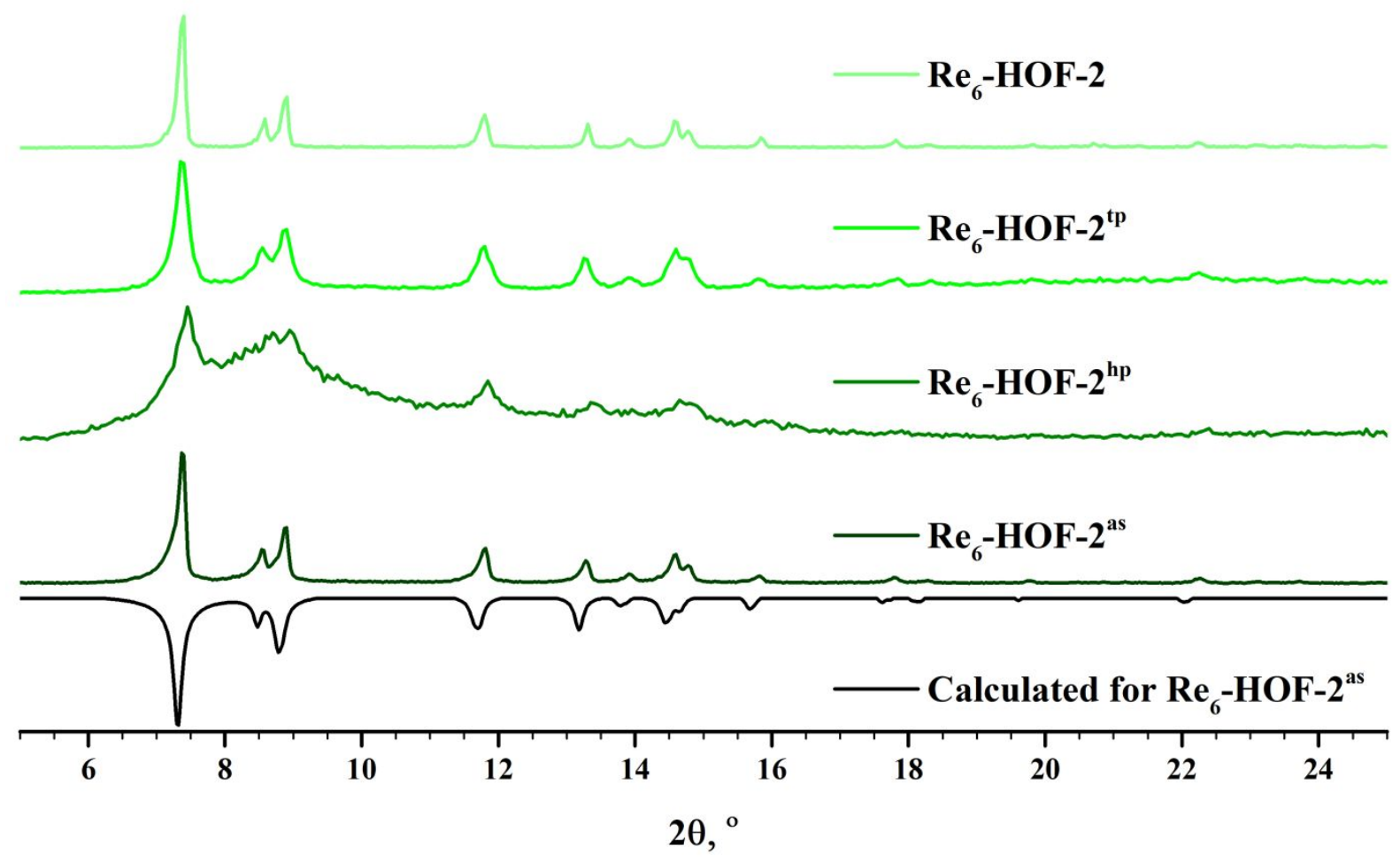

Figure S7. The X-ray powder diffraction patterns of $\mathbf{R e}_{6}-\mathrm{HOF}-\mathbf{2}^{\mathrm{as}}, \mathbf{R e}_{6}-\mathrm{HOF}-\mathbf{2}^{\mathrm{hp}}, \mathbf{R e}_{\mathbf{6}}-\mathrm{HOF}-\mathbf{2}^{\mathrm{tp}}, \mathbf{R e}_{6} \mathbf{}^{-}$ HOF-2 and the theoretical pattern generated from the unit cell parameters of $\mathbf{R e}_{\mathbf{6}}-\mathbf{H O F}-\mathbf{2}^{\text {as }}$, obtained at room temperature. 
${ }^{1} \mathrm{H}$ NMR data

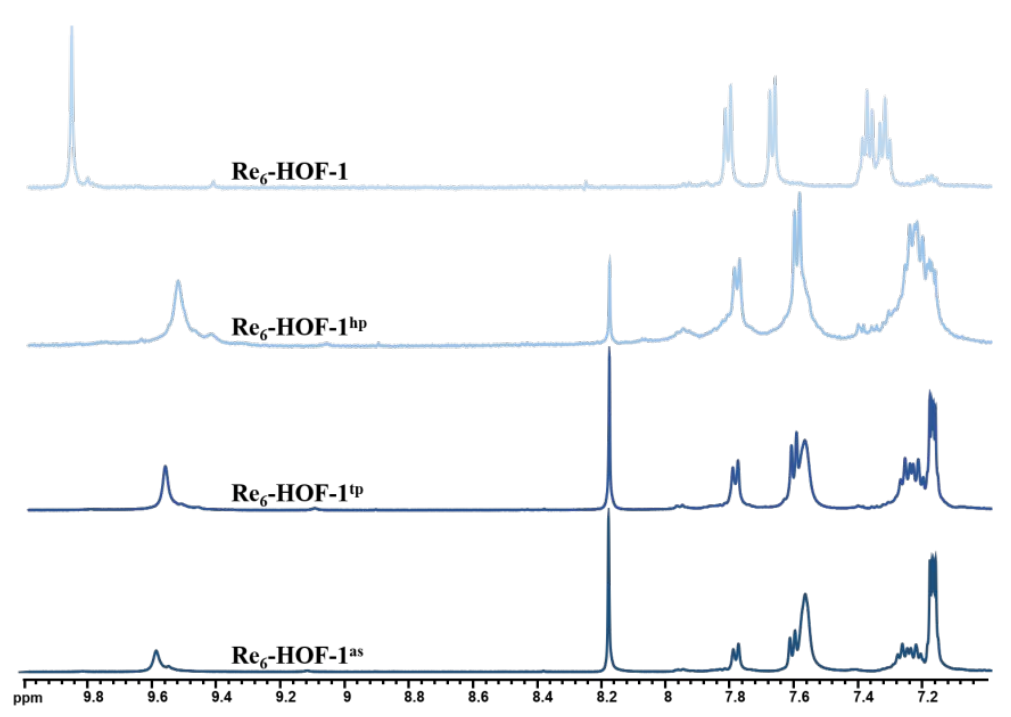

Figure S8. ${ }^{1} \mathrm{H}$ NMR spectra of $\mathbf{R e}_{6}-\mathrm{HOF}-1^{\text {as }}, \mathbf{R e}_{6}-\mathrm{HOF}-\mathbf{1}^{\mathrm{tp}}, \mathbf{R e}_{6}-\mathrm{HOF}-\mathbf{1}^{\mathrm{hp}}$ and $\mathbf{R e}_{6}-\mathrm{HOF}-1$ in DMSO-d ${ }_{6}$.

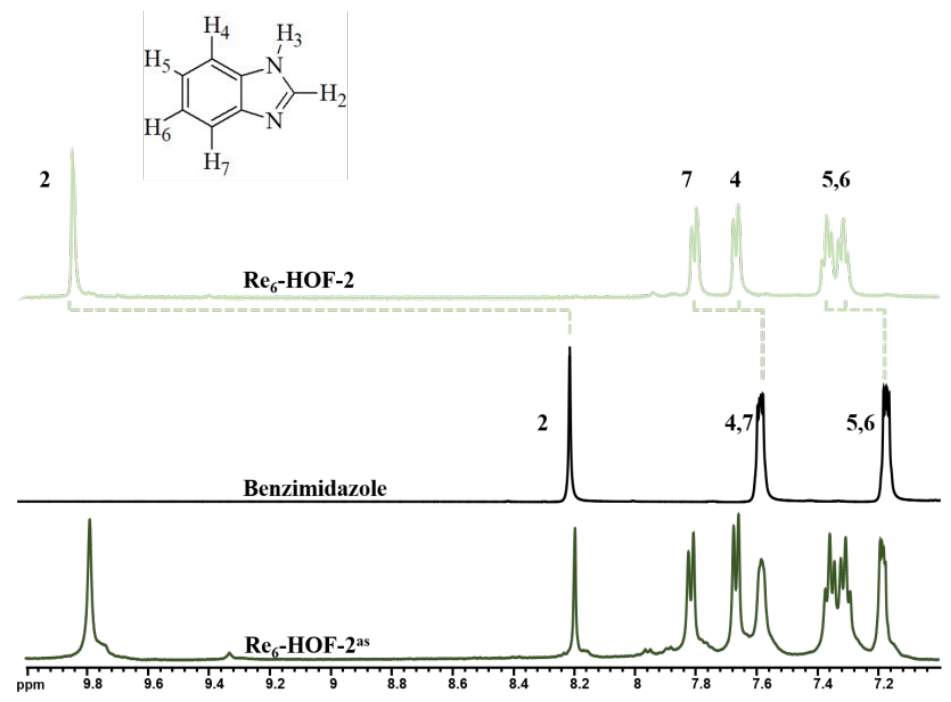

Figure S9. ${ }^{1} \mathrm{H}$ NMR spectra of $\mathbf{R e}_{6}-\mathrm{HOF}-2^{\text {as }}, \mathbf{R e}_{6}-\mathrm{HOF}-2$ and benzimidazole in DMSO-d $\mathrm{d}_{6}$.

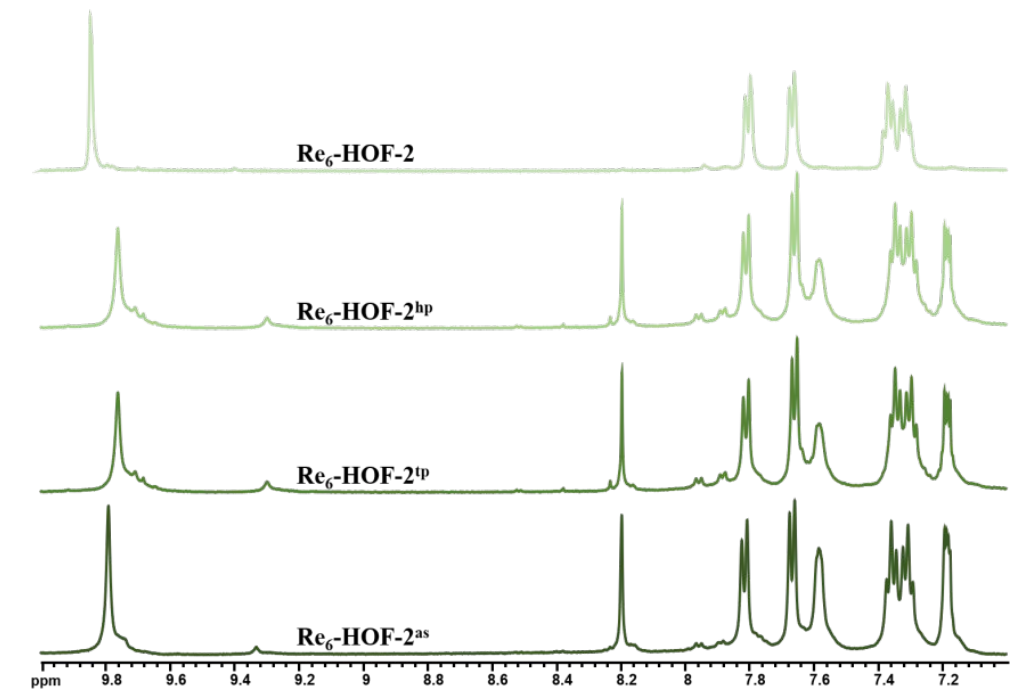

Figure S10. ${ }^{1} \mathrm{H}$ NMR spectra of $\mathbf{R e}_{6}-\mathrm{HOF}-2^{\text {as }}, \mathbf{R e}_{6}-\mathrm{HOF}-\mathbf{2}^{\mathrm{tp}}, \mathbf{R e}_{6}-\mathrm{HOF}-\mathbf{2}^{\text {hp }}$ and $\mathbf{R e}_{6}-\mathrm{HOF}-2$ in DMSO$d_{6}$. 


$\left.\right|_{2-\mathrm{C}} ^{\stackrel{m}{\infty}}$

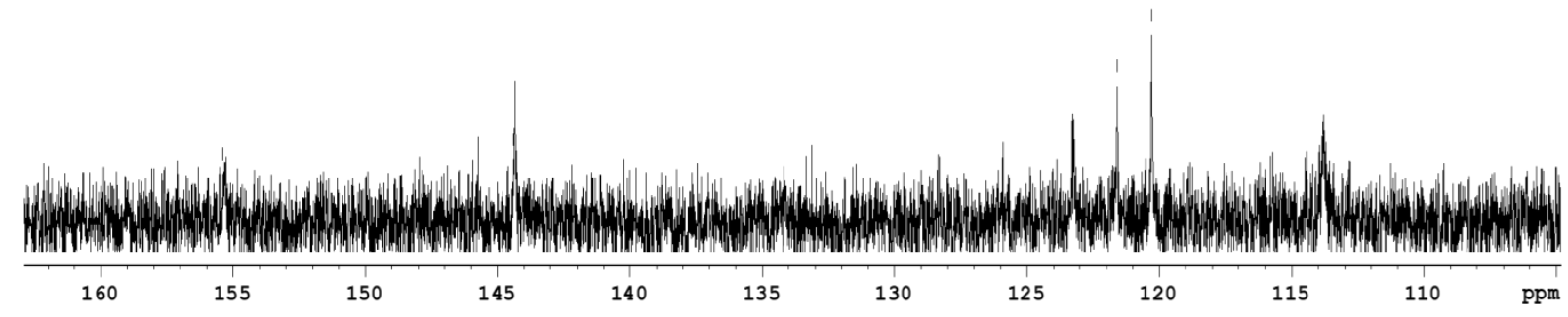

Figure S11. ${ }^{13} \mathrm{C}\left\{{ }^{1} \mathrm{H}\right\}$ NMR spectra of $\mathbf{R e}_{6}-\mathrm{HOF}-1$ in DMSO- $\mathrm{d}_{6}$.
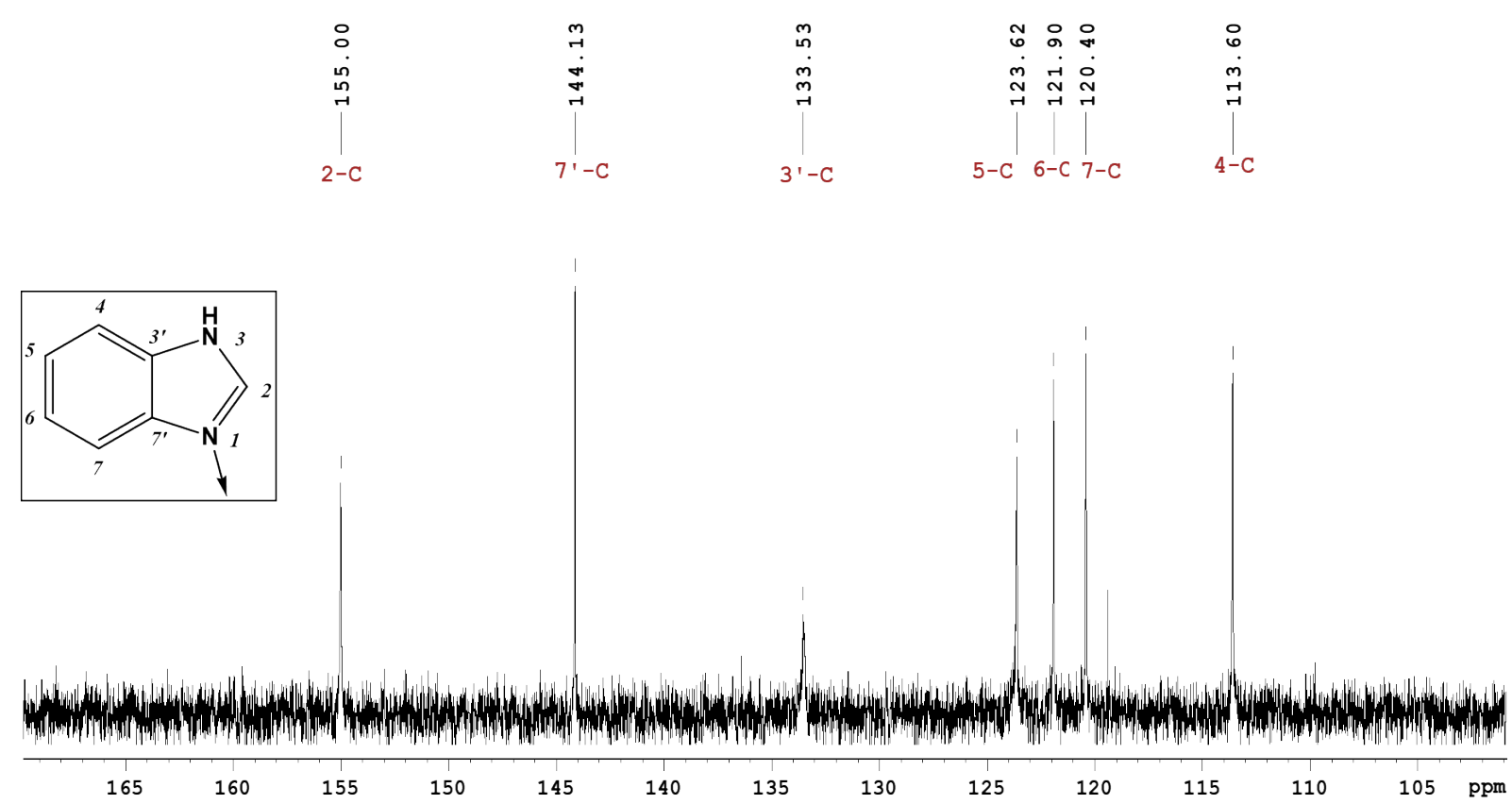

Figure S12. ${ }^{13} \mathrm{C}\left\{{ }^{1} \mathrm{H}\right\}$ NMR spectra of $\mathbf{R e}_{6}-\mathrm{HOF}-\mathbf{2}$ in DMSO-d $\mathrm{d}_{6}$. 


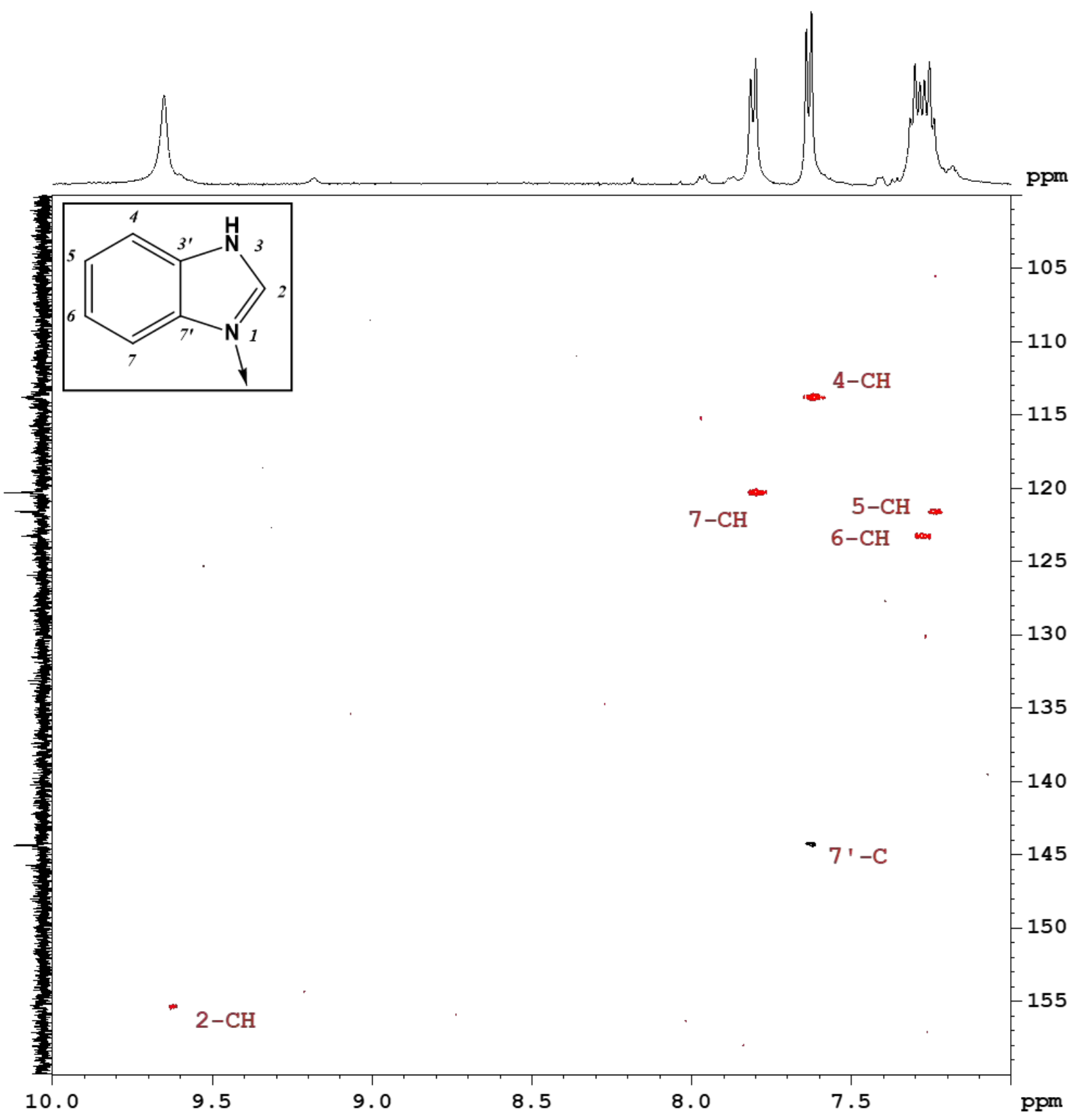

Figure S13. ${ }^{1} \mathrm{H},{ }^{13} \mathrm{C}$ HSQC (red) and HMBC (black) spectra of $\mathbf{R e}_{6}-\mathrm{HOF}-1$ in DMSO-d $\mathrm{d}_{6}$. 


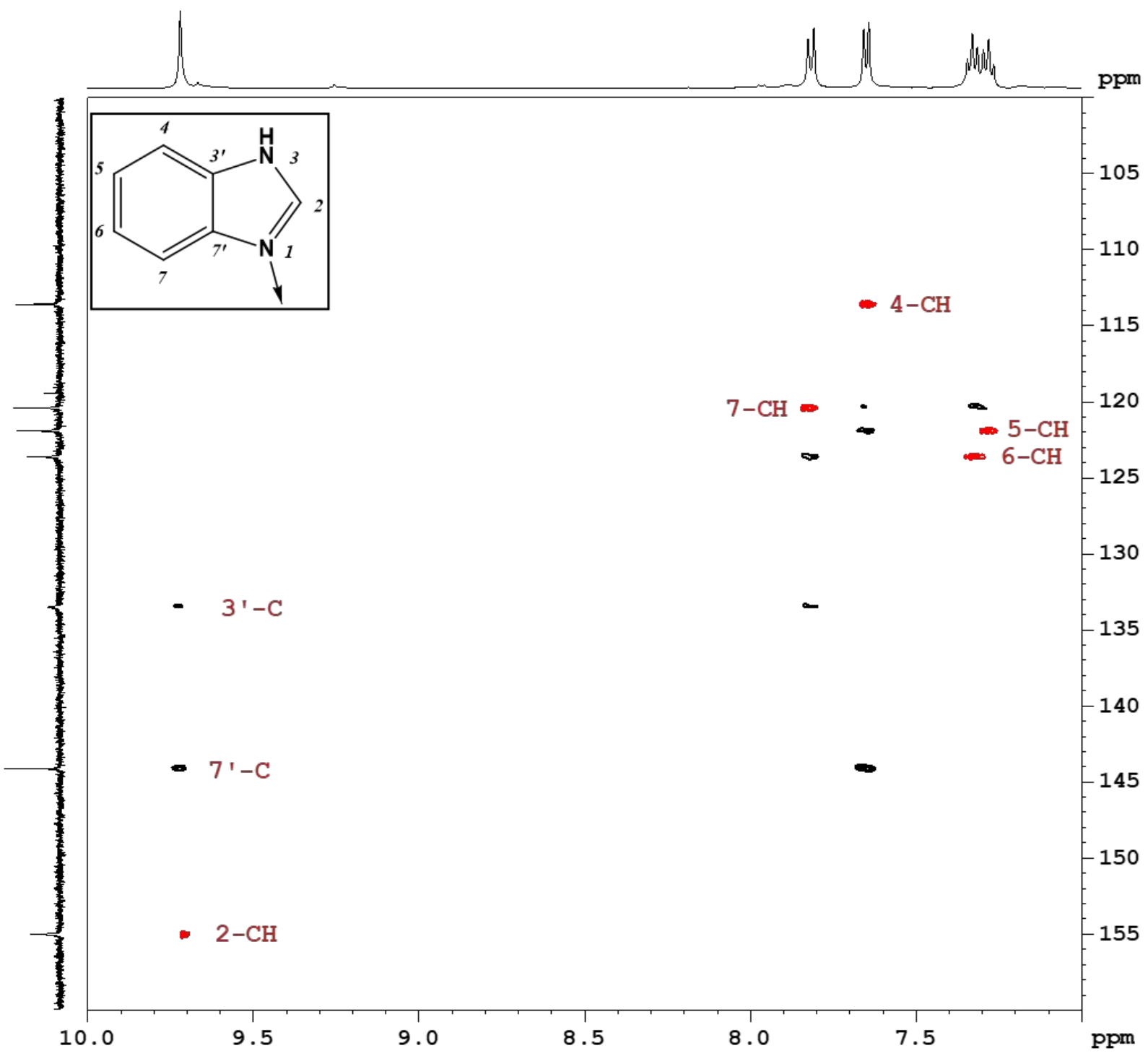

Figure S14. ${ }^{1} \mathrm{H},{ }^{13} \mathrm{C}$ HSQC (red) and HMBC (black) spectra of $\mathbf{R e}_{6}-\mathrm{HOF}-2$ in DMSO- $\mathrm{d}_{6}$. 


\section{Rieltveld refinements of powder patterns}

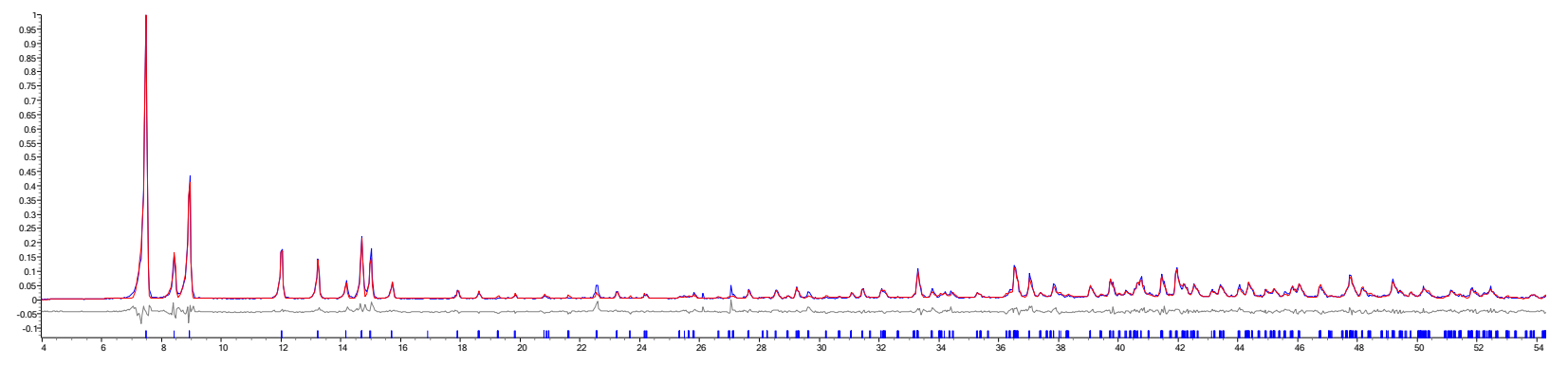

Figure S15. Rieltveld refinement (red) of $\mathbf{R e}_{6}-\mathbf{H O F - 1}{ }^{\text {as }}$ powder pattern (blue). $\mathrm{R}=9.9 \%$.

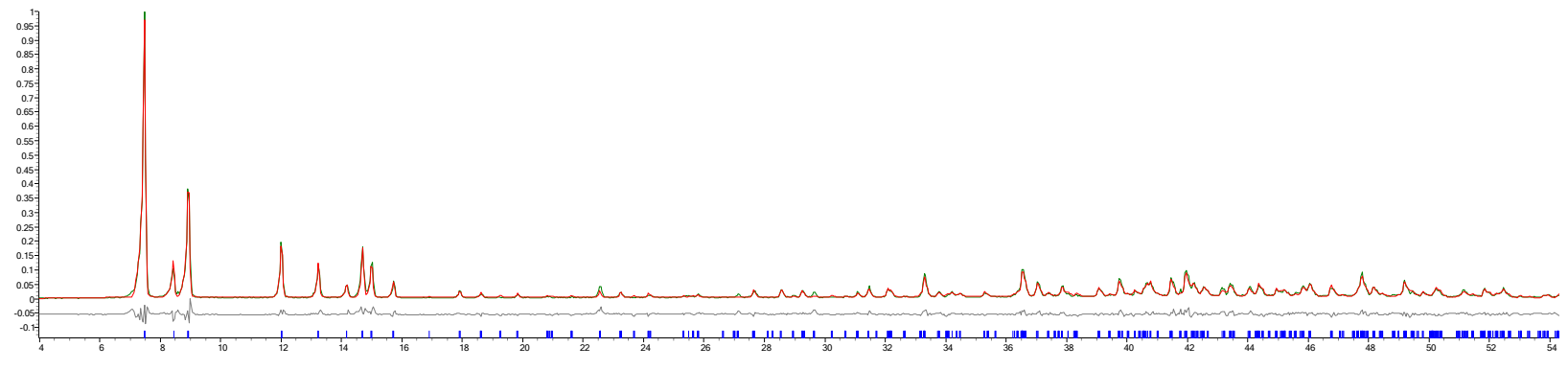

Figure S16. Rieltveld refinement (red) of $\mathbf{R e}_{6}$-HOF-1 powder pattern (green). $\mathrm{R}=10.2 \%$.

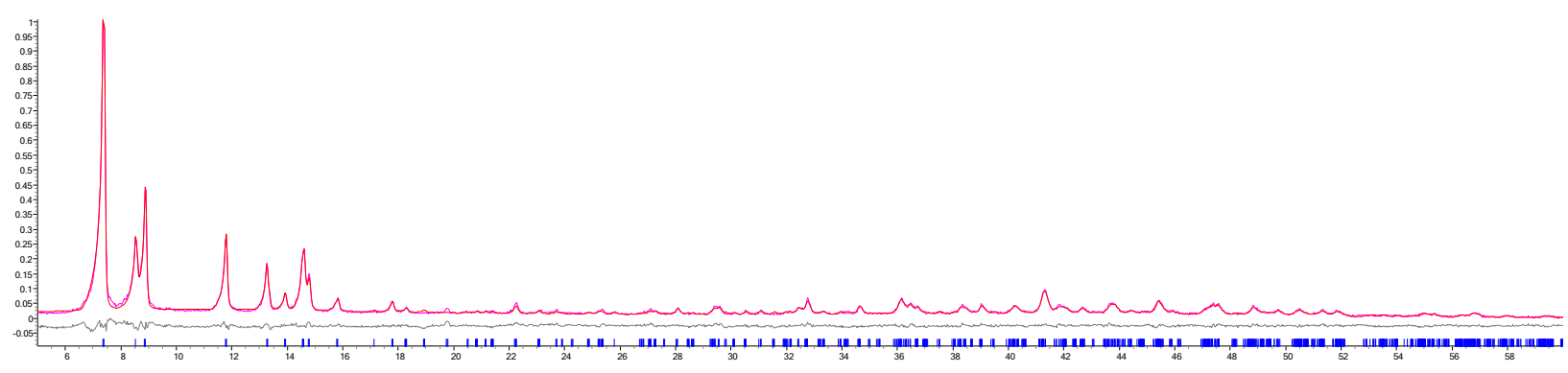

Figure S17. Rieltveld refinement (red) of $\mathbf{R e}_{6}-\mathbf{H O F}-\mathbf{2}^{\text {as }}$ powder pattern (purple). $\mathrm{R}=6.2 \%$.

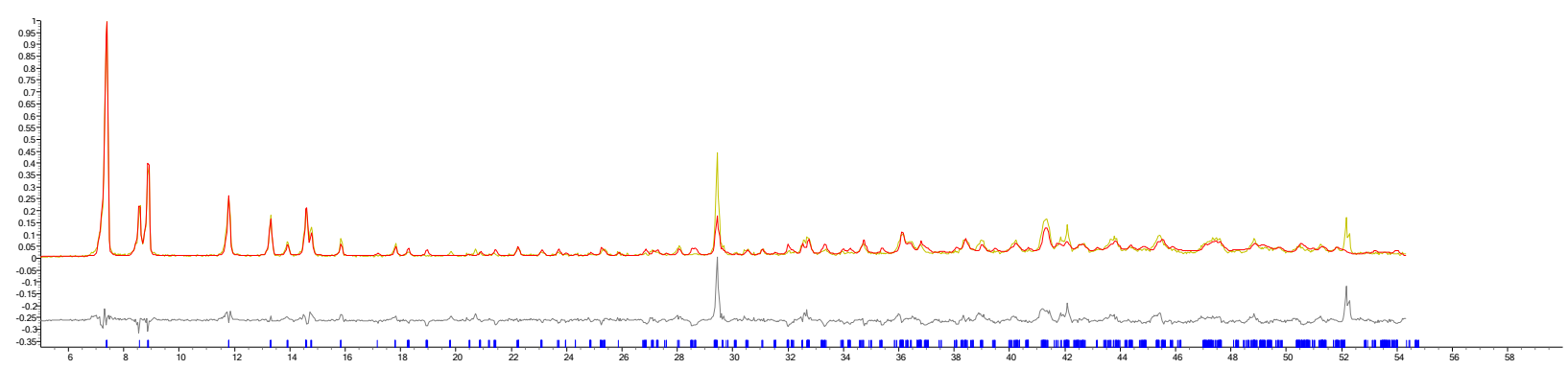

Figure S18. Rieltveld refinement (red) of $\mathbf{R e}_{6}-\mathbf{H O F - 2}{ }^{\text {as }}$ powder pattern (green). R $=23.6 \%$. Preferred-orientation of crystallites (peak at $2 \theta=29.5$ ) was observed due to which the refinement quality is low. 


\section{Sorption properties}

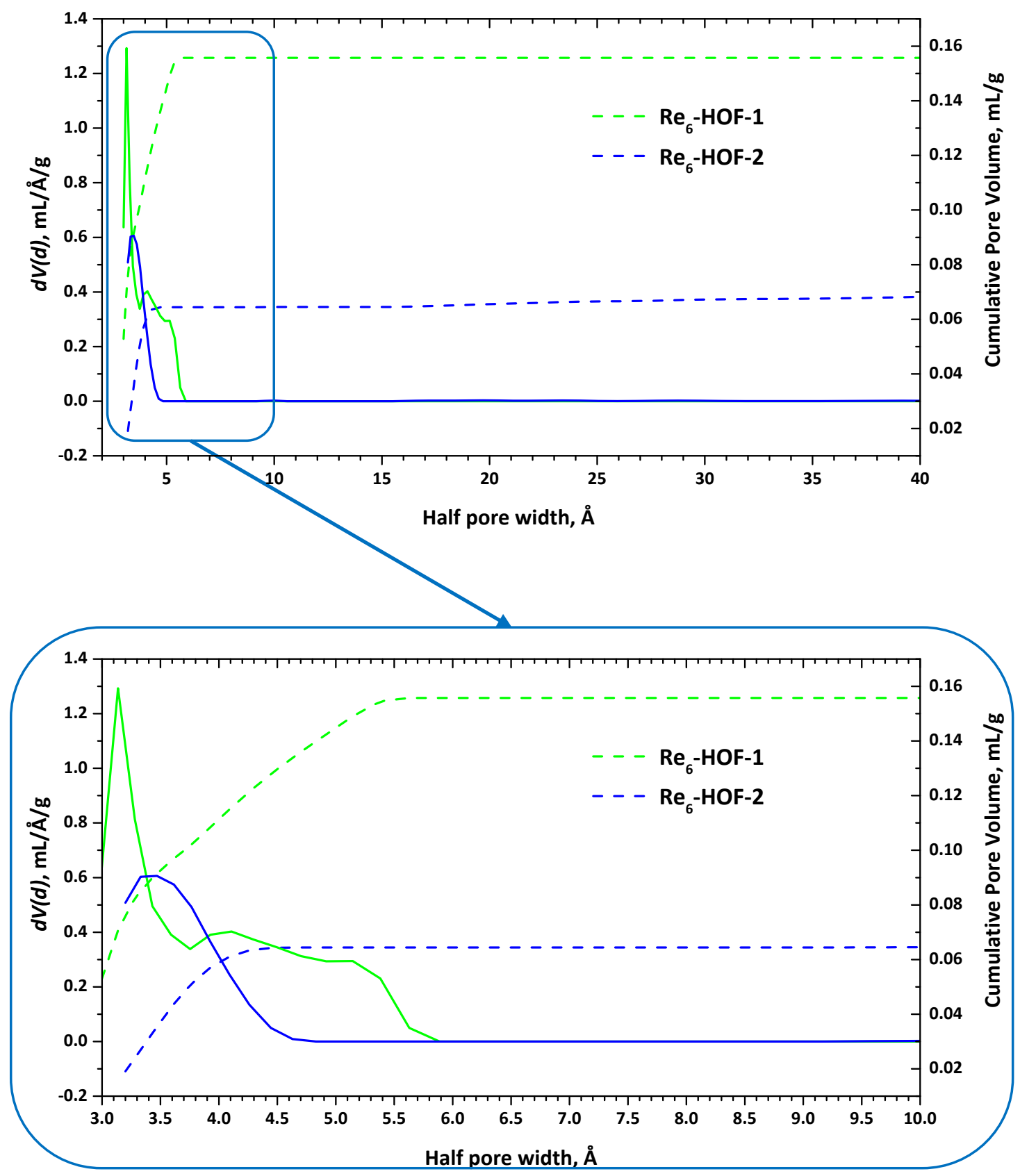

Figure S19. Calculated DFT pore size distributions (solid lines) and cumulative pore volumes (dashed lines). 


\section{Luminescence}

Table S3. Spectroscopic and photophysical parameters of cluster complexes $\mathbf{R e}_{\mathbf{6}}-\mathbf{H O F - 1}$ and $\mathbf{R e}_{\mathbf{6}}-$ HOF-2.

\begin{tabular}{|c|c|c|c|c|c|c|c|c|}
\hline \multirow{3}{*}{ Compound } & \multicolumn{5}{|c|}{ In DMSO solutions } & \multicolumn{3}{|c|}{ In solid state } \\
\hline & \multicolumn{3}{|c|}{ Aerated } & \multicolumn{2}{|c|}{ Deaerated } & \multirow[b]{2}{*}{$\lambda_{e m} / \mathrm{nm}$} & \multirow[b]{2}{*}{$\tau_{e m} / \mu \mathrm{s}$} & \multirow[b]{2}{*}{$\Phi_{e m}$} \\
\hline & $\lambda_{e m} / \mathrm{nm}$ & $\tau_{e m} / \mu \mathrm{s}(\mathrm{A})$ & $\Phi_{e m}$ & $\tau_{e m} / \mu \mathrm{s}$ & $\Phi_{e m}$ & & & \\
\hline $\mathrm{Re}_{6}$-HOF-1 & 700 & $\begin{array}{l}\tau_{1}=2.0(0.64) \\
\tau_{2}=3.7(0.36)\end{array}$ & 0.03 & 15.9 & 0.16 & 700 & $\begin{array}{c}\tau_{1}=1.1(0.36) \\
\tau_{2}=4.9(0.47) \\
\tau_{3}=10.7(0.17)\end{array}$ & 0.10 \\
\hline $\mathrm{Re}_{6}-\mathrm{HOF}-2$ & 700 & $\begin{array}{l}\tau_{1}=2.4(0.89) \\
\tau_{2}=4.6(0.11)\end{array}$ & 0.03 & 15.8 & 0.15 & 700 & $\begin{array}{c}\tau_{1}=0.8(0.35) \\
\tau_{2}=3.9(0.45) \\
\tau_{3}=13.0(0.20)\end{array}$ & 0.08 \\
\hline
\end{tabular}

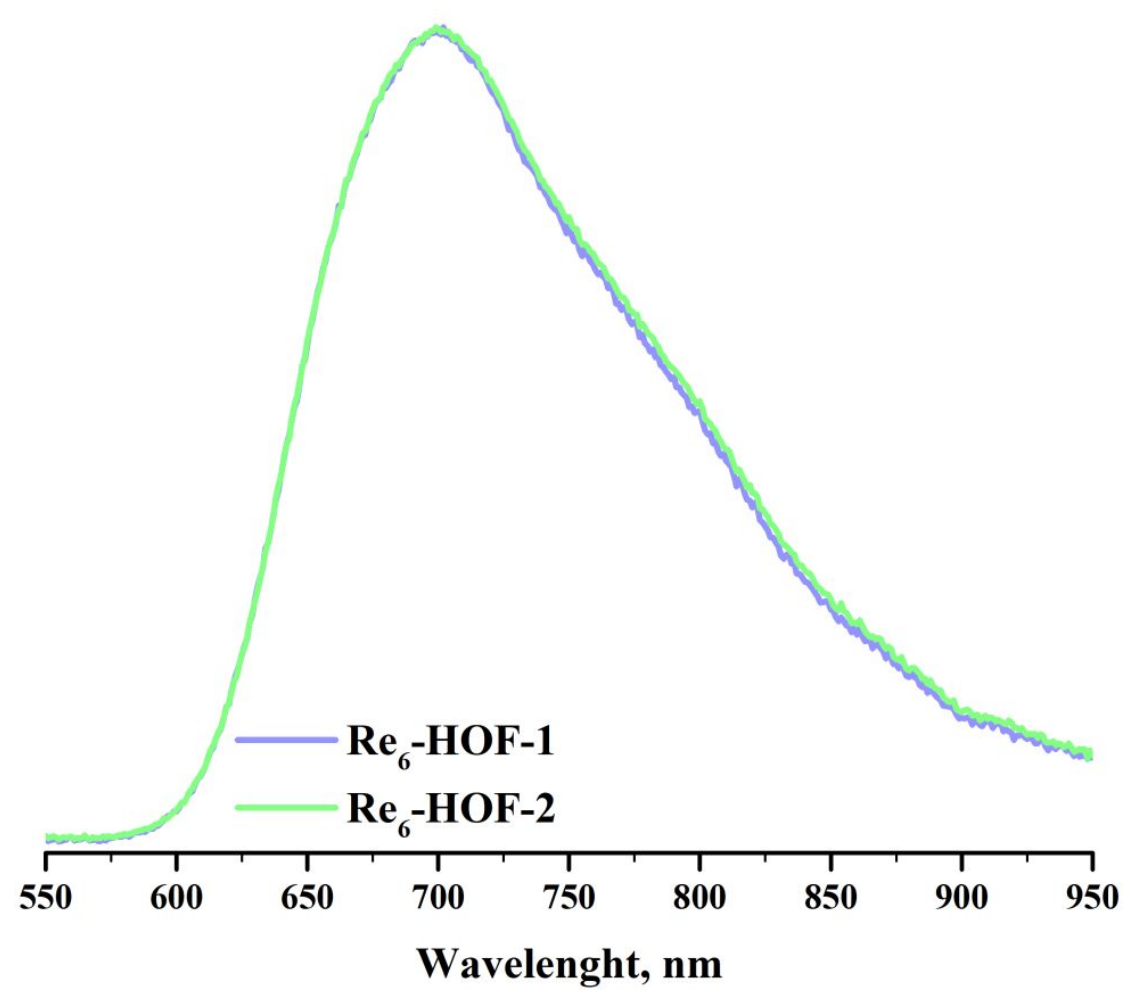

Figure S20. Normalized emission spectra of $\mathbf{R e}_{6}-\mathrm{HOF}-\mathbf{1}$ and $\mathbf{R e}_{6}-\mathrm{HOF}-\mathbf{2}$ in aerated DMSO. 


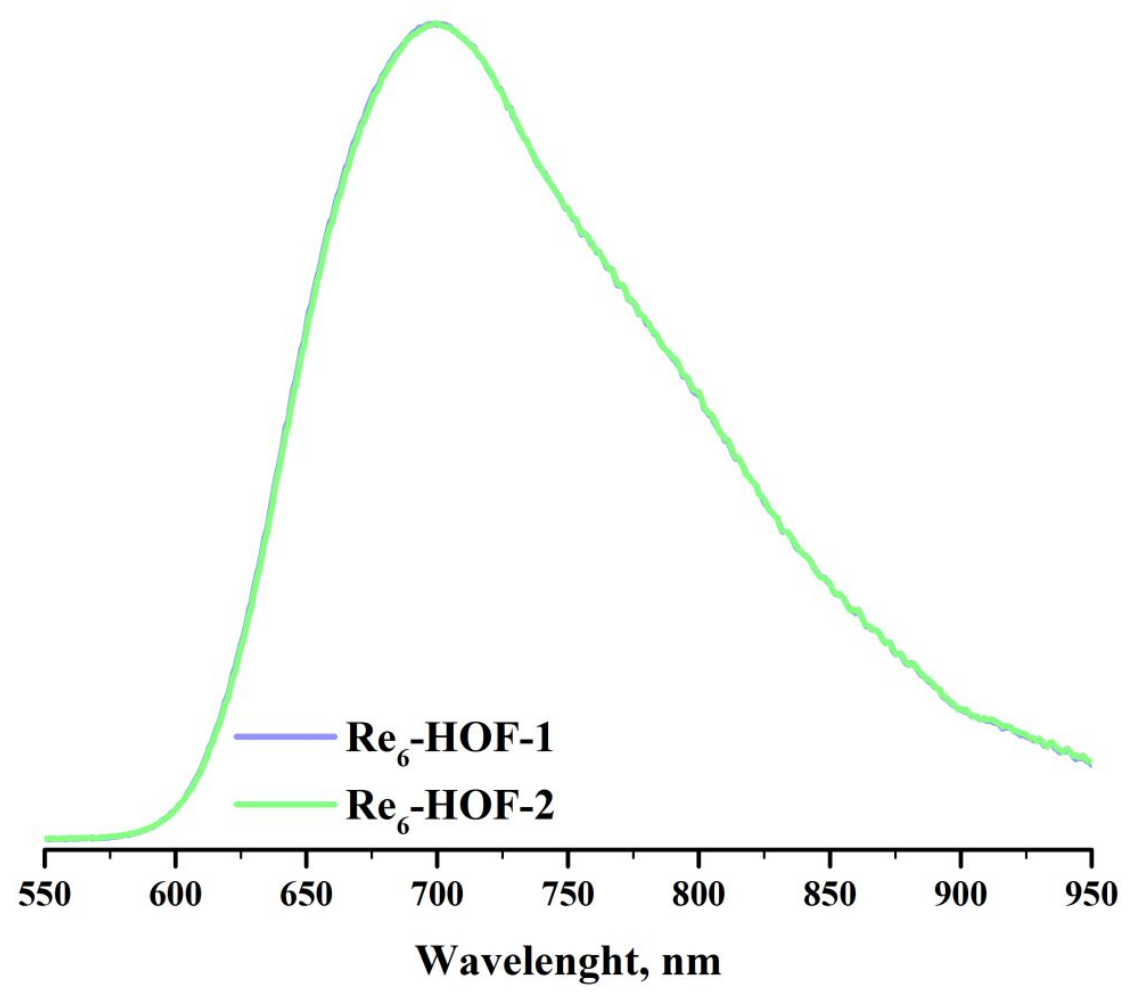

Figure S21. Normalized emission spectra of $\mathbf{R e}_{6}-\mathrm{HOF}-\mathbf{1}$ and $\mathbf{R e}_{6}-\mathrm{HOF}-\mathbf{2}$ in deaerated DMSO.
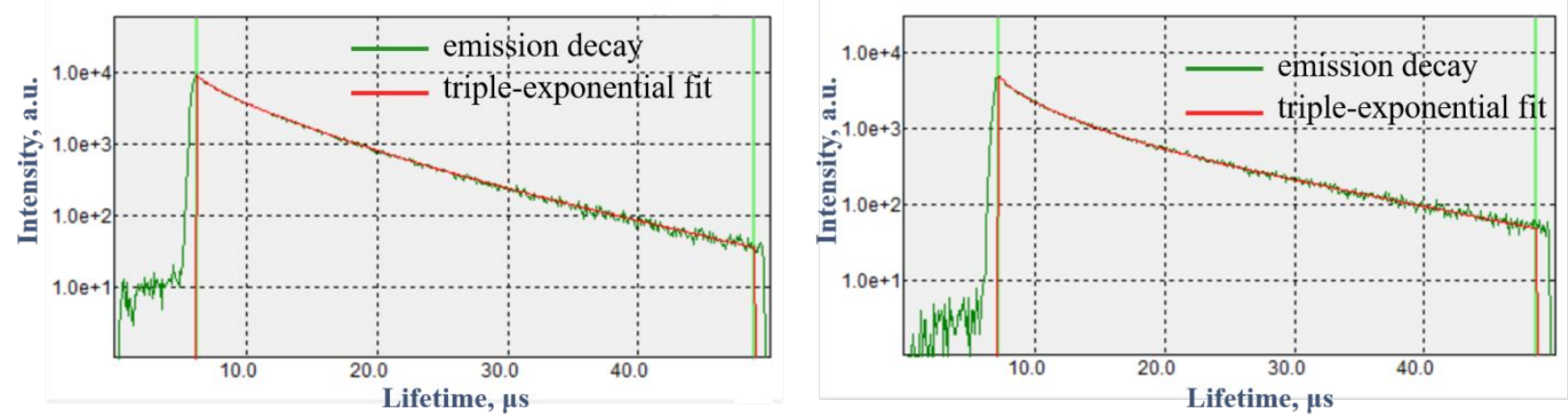

Figure S22. Luminescence decay curves of $\mathbf{R e}_{6}-\mathrm{HOF}-\mathbf{1}$ (left) and $\mathbf{R e}_{6}$-HOF-2 (right)in solid state.
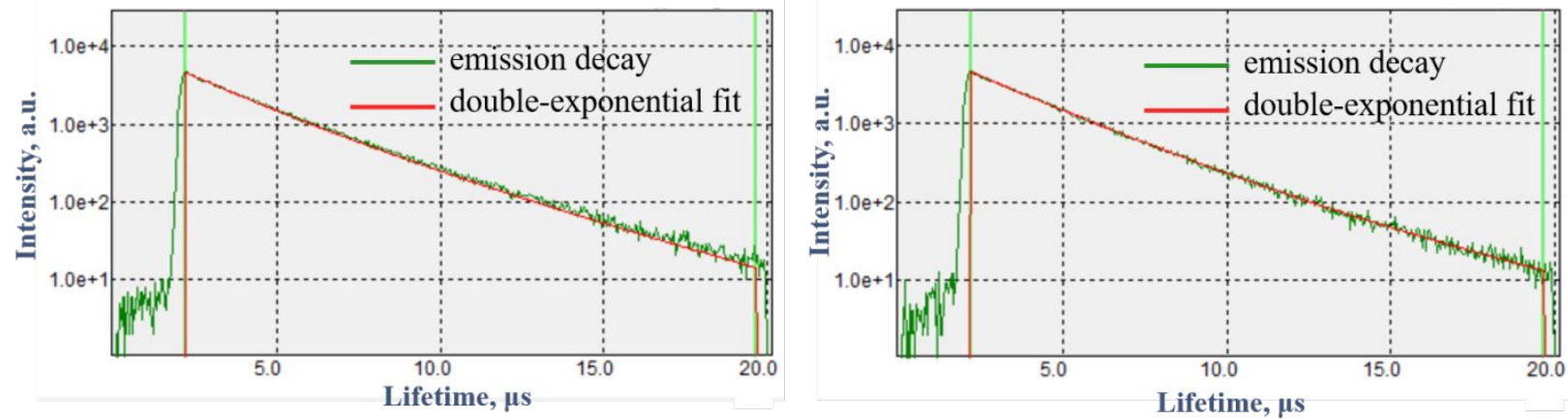

Figure S23. Luminescence decay curves of $\mathbf{R e}_{6}-\mathrm{HOF}-\mathbf{1}$ (left) and $\mathbf{R e}_{6}-\mathrm{HOF}-\mathbf{2}$ (right) in aerated DMSO. 

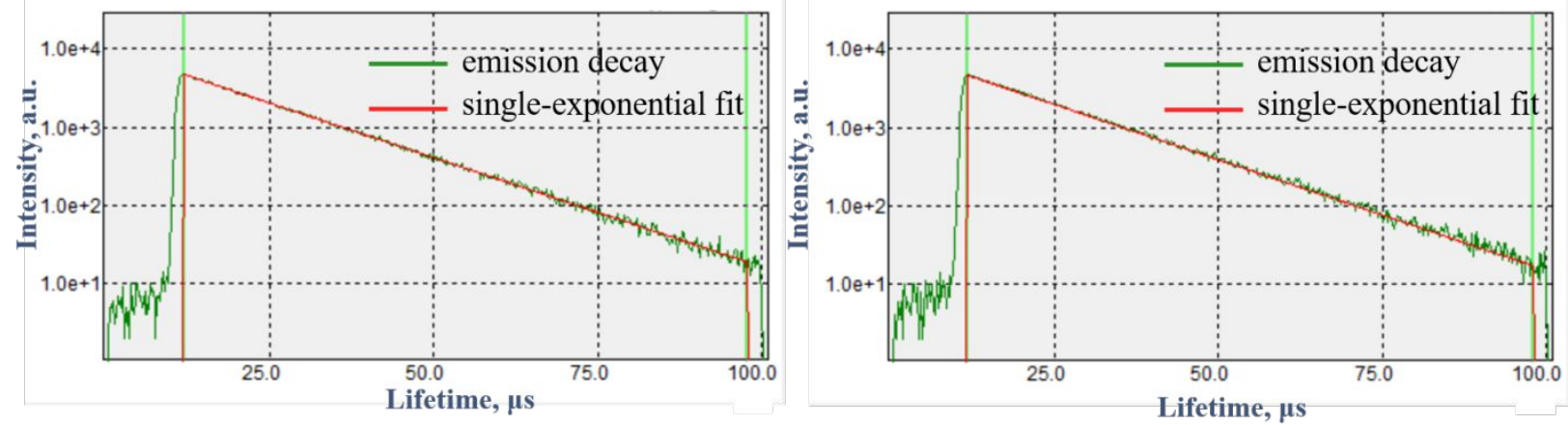

Figure S24. Luminescence decay curves of $\mathbf{R e}_{6}-\mathrm{HOF}-\mathbf{1}$ (left) and $\mathbf{R e}_{6}-\mathrm{HOF}-\mathbf{2}$ (right) in deaerated DMSO. 


\section{References}

1. Konovalov, D.I., et al., Synthesis and luminescence properties of apically homoleptic octahedral rhenium clusters with pyrazole and 3,5-dimethylpyrazole. Inorg. Chim. Acta, 2019. 498: p. 119128.

2. Konovalov, D.I., et al., Octahedral chalcogenide rhenium cluster complexes with imidazole. Polyhedron, 2019. 165: p. 79-85.

3. Konovalov, D.I., et al., Water-soluble rhenium clusters with triazoles: the effect of chemical structure on cellular internalization and the DNA binding of the complexes. Chem. Eur. J., 2020. 26(61): p. 13904-13914. 